\title{
The Newton Fractal's Leonardo Sequence Study with the Google Colab
}

\author{
Francisco Regis Vieira Alves ${ }^{1 *}$, Renata Passos Machado Vieira ${ }^{1}$ \\ ${ }^{1}$ Federal Institute of Science and Technology of Ceara - IFCE, BRAZIL \\ *CORRESPONDENCE: $\square$ fregis@ifce.edu.br
}

\begin{abstract}
The work deals with the study of the roots of the characteristic polynomial derived from the Leonardo sequence, using the Newton fractal to perform a root search. Thus, Google Colab is used as a computational tool to facilitate this process. Initially, we conducted a study of the Leonardo sequence, addressing it fundamental recurrence, characteristic polynomial, and its relationship to the Fibonacci sequence. Following this study, the concept of fractal and Newton's method is approached, so that it can then use the computational tool as a way of visualizing this technique. Finally, a code is developed in the Phyton programming language to generate Newton's fractal, ending the research with the discussion and visual analysis of this figure. The study of this subject has been developed in the context of teacher education in Brazil.
\end{abstract}

Keywords: Newton's fractal, Google Colab, Leonardo sequence, visualization

\section{INTRODUCTION}

Interest in the study of homogeneous, linear and recurrent sequences is becoming more and more widespread in the scientific literature. Therefore, the Fibonacci sequence is the best known and studied in works found in the literature, such as Oliveira and Alves (2019), Alves and Catarino (2019). In these works, we can see an investigation and exploration of the Fibonacci sequence and the complexification of its mathematical model and the generalizations that result from it. However, new sequences are being introduced, thus having a few papers published in international journals. As an example of this, we have recently introduced Leonardo sequence in the literature, still with a considerable shortage of works in pure mathematics and mathematics teaching.

However, based on the work of Catarino and Borges (2019, in press), we will conduct a study of these Leonardo numbers, investigating and discussing the study of the roots of their respective characteristic polynomial. There are many mathematical and numerical methods to perform these calculations, so in this work the Newton method will be used as a way to obtain a search and determination of the roots of this recurring sequence, still working on the analysis of its generated fractal. For this, it is necessary to use some computational resources, and therefore, Google Colab was selected as a tool that facilitates the teaching process for this research for free and that allows us to explore the visualization.

Google Colab, also known as Collaboratory, is increasingly being used in education and education, as this project aims to disseminate machine learning education and research (Randlesi Pasquetto, Golshan, \& Borgman, 2017). In this feature, you can add notebooks, and you can then comment out the code as if it were a kind of Google Docs (Google text editing tool). Besides, users are allowed to collaborate and share these blocks and assist in the development of code developed in the Phyton programming language.

Article History: Received 4 September $2019 \bullet$ Revised 5 November $2019 \bullet$ Accepted 5 November 2019

(C) 2020 by the authors; licensee Modestum Ltd., UK. Open Access terms of the Creative Commons Attribution 4.0 International License (http://creativecommons.org/licenses/by/4.0/) apply. The license permits unrestricted use, distribution, and reproduction in any medium, on the condition that users give exact credit to the original author(s) and the source, provide a link to the Creative Commons license, and indicate if they made any changes. 
Finally, the following sections will be discussed in the recurring Leonardo sequence, Newton's method, and then fractal theory involving an application of Google Colab as a computational resource for plotting Newton's fractal of these numbers, and their corresponding visualization.

\section{THE LEONARDO SEQUENCE}

Leonardo's recurring non-homogeneous sequence, which we shall denote by $\left\{L e_{n}\right\}_{n \in I N}$ is a linear and recurrent sequence, having its characteristic recurrence formula defined as:

$$
L e_{n}=L e_{n-1}+L e_{n-2}+1, n \geq 2
$$

with $L e_{n}$ the nth term of the Leonardo sequence and the initial terms indicated by $L e_{0}=L e_{1}=1$.

Therefore, we have the first numerical terms of this sequence as: 1,1,3,5,9,15,25,41,67,109, 177. Immediately, according to the work of Catarino \& Borges (2019, in press), We thus verify that all the terms of this sequence are odd. The authors also performed algebraic manipulations on this recurrence, obtaining a new homogeneous recurrence. Thus, from the recurrence relationship seen in equation 1, we can substitute, resulting in:

$$
L e_{n+1}=L e_{n}+L e_{n-1}+1
$$

Now by subtracting the expressions indicated in (1) - (2), we have:

$$
\begin{aligned}
& L e_{n}-L e_{n+1}=L e_{n-1}+L e_{n-2}+1-\left(L e_{n}+L e_{n-1}+1\right) \\
& L e_{n}-L e_{n+1}=L e_{n-2}-L e_{n} \\
& L e_{n+1}=2 L e_{n}-L e_{n-2}, n \geq 2 .
\end{aligned}
$$

Leonardo's numbers also have a relation with the emblematic sequence of Fibonacci, thus existing a recurrence relation with characteristics of these two sequences. Thus, remembering the formula for obtaining Fibonacci numbers $F_{n}=F_{n-1}+F_{n-2}, n \geq 2$, with the initial values defined by $F_{0}=F_{1}=1$, obtaining the following numerical elements: $1,1,2,3,5,8,13,21,34,55$.

Proposition 1. For every positive integer $n \geq 0$, we have that the relation of Leonardo numbers with Fibonacci numbers is given by (Catarino \& Borges, 2019 in press):

$L e_{n}=2 F_{n+1}-1, n \geq 0$.

Proof. Using the principle of mathematical induction, we immediately have that:

To initial value $n=0$, we can determine that

$1=L e_{0}=1=2 \cdot 1-1=2 \cdot F_{1}-1$.

So let's assume the property is valid for every positive integer $n=k, k \in I N$.

$$
\begin{aligned}
& L e_{k}=L e_{k-1}+L e_{k-2}+1 \\
& L e_{k}=\left(2 F_{k}-1\right)+\left(2 F_{k-1}-1\right)+1 \\
& L e_{k}=2\left(F_{k}+F_{k-1}\right)-1 \\
& L e_{k}=2 F_{k+1}-1
\end{aligned}
$$

Performing the suitable substitutions and using the inducement hypothesis, it follows that $n=k+1$.

$$
\begin{aligned}
& L e_{k+1}=L e_{k}+L e_{k-1}+1 \\
& L e_{k+1}=\left(2 F_{k+1}-1\right)+\left(2 F_{k}-1\right)+1 \\
& L e_{k+1}=2\left(F_{k+1}+F_{k}\right)-1 \\
& L e_{k+1}=2 F_{k+2}-1
\end{aligned}
$$

Henceforth we will establish the characteristic polynomial of the Leonardo sequence from the fundamental recurrence $L e_{n}=2 L e_{n-1}-L e_{n-2}$. With this, we will have the following quotients:

$$
\begin{aligned}
& L e_{n}=2 L e_{n-1}-L e_{n-2} \\
& \frac{L e_{n+1}}{L e_{n}}=2 \frac{L e_{n}}{L e_{n}}-\frac{L e_{n-2}}{L e_{n}} \cdot 1 \\
& \frac{L e_{n+1}}{L e_{n}}=2-\frac{L e_{n-2}}{L e_{n}} \cdot \frac{L e_{n-1}}{L e_{n-1}}
\end{aligned}
$$

Studying the relationship of convergence between this sequence and the behavior of the quotient of Leonardo numbers $\frac{\boldsymbol{L} \boldsymbol{e}_{n+1}}{\boldsymbol{L} \boldsymbol{e}_{n}}$, we can then establish a subsequence given by dividing a Leonardo term by its 
Table 1. Convergence analysis between Leonardo's neighboring terms. Prepared by the authors

\begin{tabular}{ccc}
\hline $\boldsymbol{n}$ & $\boldsymbol{L} \boldsymbol{e}_{\boldsymbol{n}}$ & $\frac{\boldsymbol{L e}_{\boldsymbol{n + 1}}}{\boldsymbol{L e}_{\boldsymbol{n}}}$ \\
\hline 0 & 0 & $\nexists$ \\
\hline 1 & 1 & 1 \\
\hline 2 & 1 & 3 \\
\hline 3 & 3 & 1.66 \\
\hline 4 & 5 & 1.8 \\
\hline 5 & 9 & 1.66 \\
\hline 6 & 15 & 1.66 \\
\hline 7 & 25 & 1.64 \\
\hline 8 & 41 & 1.63 \\
\hline 9 & 67 & 1.62 \\
\hline 10 & 109 & 1.62 \\
\hline 11 & 177 & 1.62 \\
\hline 12 & 287 & 1.62 \\
\hline 13 & 465 & 1.61 \\
\hline 14 & 753 & 1.61 \\
\hline 15 & 1219 & 1.61 \\
\hline
\end{tabular}

successor, converging to the real value 1.61. This number is known from the scientific literature as the gold number (see Table 1). It is noteworthy that this relationship is the same as that for the Fibonacci sequence.

Thus, we have the following limit indicated by $\lim _{n \rightarrow \infty} \frac{L e_{n+1}}{L e_{n}} \approx 1.61=\phi \in I R$. Therefore, returning to the previous equation, we will make the appropriate substitutions as follows:

$$
\begin{aligned}
& \frac{L e_{n+1}}{L e_{n}}=2 \frac{L e_{n}}{L e_{n}}-\frac{L e_{n-2}}{L e_{n}} \frac{L e_{n-1}}{L e_{n-1}} \\
& \phi=2-\frac{1}{\frac{L e_{n-1}}{L e_{n-2}} \cdot \frac{L e_{n}}{L e_{n-1}}} \\
& \phi=2-\frac{1}{\phi^{2}} \\
& \phi^{3}=2 \phi-1
\end{aligned}
$$

Given this, we establish the following characteristic polynomial of the Leonardo sequence $\phi^{3}-2 \phi^{2}+1=0$ having three roots, whose behavior will be analyzed in the following sections according to numerical methods and with the help of a collaborative computational tool easily accessible on the internet.

\section{THE NEWTON'S FRACTAL WITH THE GOOGLE COLAB}

There are many methods for obtaining the roots of a mathematical equation, varying according to their degree, so the best known is the classic Baskhara method. For equations of higher-order to the fourth order, Niels Henrik Abel (1802 - 1829) proved that it is not possible to obtain the roots analytically through formulas and coefficient combinations (Cláudio \& Martins, 2000). Thus, numerical methods and computational resources are used to obtain these roots from a rough and/or qualitative approach.

A well-known method that will be used in this work is Newton's method, which performs the calculation of successive approximations of polynomial zeros, and can quickly and qualitatively investigate the iterations beginning near the desired root. However, there are cases in which the iteration starts far from the desired root, being careful to avoid systematic errors in the use of this method (Teodoro \& Aguilar, 2015).

According to Lima (1976), a linear and recurrent sequence has a recurrence formula and carries infinite and enumerable values of terms. Moreover, every sequence of this type still has a characteristic polynomial, varying according to its order, and therefore recurring from the analytical formula: $x_{n+3}+r x_{n+2}+p x_{n+1}+$ $q x_{n}=0$, where $r, p, q \in Z$ and $q \neq 0$ or $p \neq 0$. For the particular case $q=0$ or $p=0$ the sequence will be first or second order (Zierler, 1959).

Thus, it is necessary to know the roots of its characteristic polynomial, also called the characteristic equation of the sequence. Therefore, one of the ways to obtain these roots is through the Newton method, 
where this equation is transformed into a function, and thus a search is made for its roots and its corresponding graph is constructed.

This method was developed by Isaac Newton and Joseph Raphson to estimate the roots of a function by approximations, using the formula $N\left(x_{n+1}\right)=x_{n}-\frac{f\left(x_{n}\right)}{f^{\prime}\left(x_{n}\right)}$, where $x_{0}$ is the initial approximation, the nth iteration of the method $f^{\prime}\left(x_{n}\right)$ and the derivative of the function $f\left(x_{n}\right)$ (Macleiod, 1984). Later this method will be used to obtain the roots of the Leonardo sequence, analyzing and discussing them by visualizing certain properties.

In the late nineteenth century, many mathematicians were concerned with applications of the classical methods of mathematical functions, because when they were not regular, this function was ignored. However, this attitude changed with the discovery of non-soft objects, providing a representation of many natural phenomena than the figures of classical geometry. According to Macedo (2015):

Fractal objects have a very complex structure to be studied by the classical methods of analytical geometry and calculus, to study them requires the definition of new concepts, such as Hausdorff's dimension, and the use of new areas of mathematics. Among these new areas are fractional calculus and deformed derivatives, in recent years there has been a very large growth in the use of fractional calculus and deformed derivatives for the study of functions and problems defined in fractal domains (Macedo, 2015, p. 11).

A fractal is generated from an iteratively applied, dynamically applied mathematical formula producing fascinating results corresponding to certain phenomena. Fractals can, in turn, take on a multitude of shapes and thus present difficult conclusions or forms of systematic analysis, derived from the regularity of identification, for example, of $2 \mathrm{D}$ or $3 \mathrm{D}$ geometric patterns.

According to Carreira and Andrade (1999) fractals are characterized by having at least one of the following properties: i) self-similarity: presenting a piece of the fractal similar to the rest of the figure; ii) infinite complexity: presents an infinite number of interactions in the fractal; iii) fractal dimension: the dimension can be fractional.

These fractals can be applied and analyzed according to the modeling of their objects, making a comparison with their fractal geometry, besides considering several approximation factors (Burton, 2009). Being applied in several areas of knowledge, including engineering and science, we highlight its application in the area of mathematics, being possible to analyze and study the roots of the characteristic polynomials of linear and recurrent sequences.

Newton's fractal, therefore, is nothing more than the junction of Newton's method and fractal theory, transforming mathematical functions into media, which can be figures, music and dynamic animations. Google Colab, in turn, is a free Google-hosted tool running in the cloud. Using a programming language in Phyton, ease of configuration and some libraries already installed, this feature was then selected for this work as a way of teaching the recurring sequence of Leonardo.

In the next section, Newton's fractal of the Leonardo sequence is then generated through Google Colab, thus teaching these numbers and analyzing the roots of their characteristic polynomial.

\section{THE FRACTAL STUDY ABOUT THE LEONARDO SEQUENCE AND THE VISUALIZATION}

The characteristic polynomials of a linear sequence is important in determining some properties, such as obtaining the Binet formula among others. Thus, given the characteristic equation of the Leonardo sequence that we indicated in the previous section $\left(\phi^{3}-2 \phi^{2}+1=0\right)$, obtained in the section above, we have the existence of three roots, denoted by $\alpha, \beta, \gamma$. Using Newton's method, we can then graph the function; it is possible to observe in Figure 1 the existence of three real roots $\alpha, \beta, \gamma \in I R$. Therefore, one is equal to 1 ( $\alpha=$ 1 ), one negative in the range [-1,0] and the other positive in the range [1,5,2]. Remember that this interval is important for the initial kick of the numerical method that will be applied. Keeping in mind that the closer to the root the initial value, the fewer iterations there will be, the faster the exact root value of the function will be. However, it is important to analyze the $2 \mathrm{D}$ graph so that this value is then set, starting the root search. 


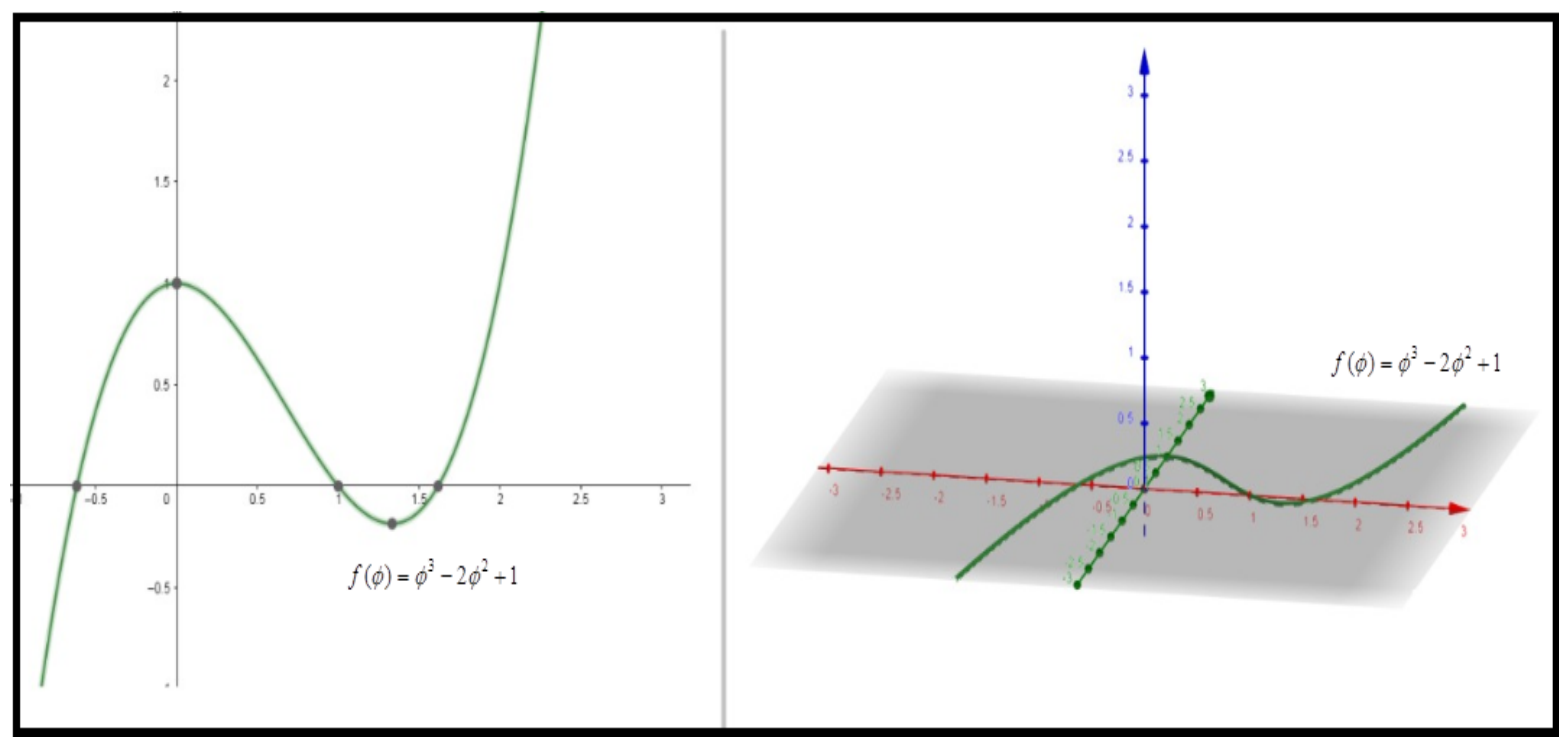

Figure 1. The graph of the Leonardo sequence function. Prepared by the authors

Following the application of Newton 's method, we have the function $f(\phi)=\phi^{3}-2 \phi^{2}+1$ :. Therefore, using the formula, we further verify that:

$$
N\left(\phi_{n+1}\right)=\phi_{n}-\left(\frac{\phi_{n}{ }^{3}-2 \phi_{n}{ }^{2}+1}{3 \phi_{n}{ }^{2}-4 \phi_{n}}\right)=\frac{2 \phi_{n}{ }^{3}-2 \phi_{n}{ }^{2}-1}{3 \phi_{n}{ }^{2}-4 \phi_{n}}
$$

Given this, we seek to find the two real roots not previously discussed. Starting the search for the negative root, we have to, assuming the initial value of $\beta_{0}=-1$. Then we check:

$$
\begin{aligned}
& \beta_{0}=-1 \\
& \beta_{1}=-0.7142857142857143 \\
& \beta_{2}=-0.0 .626578073089701 \\
& \beta_{3}=-0.6181106971045955 \\
& \beta_{4}=-0.6180339950172198 \\
& \beta_{5}=-0.6180339887498949
\end{aligned}
$$

From the fourth iteration a great precision of the decimal places is noted, thus obtaining the negative root $\beta=-0.6180$. Finally, looking for the other positive real root $(\gamma)$ of the equation $\phi^{3}-2 \phi^{2}+1=0$, we will assume the initial value $\gamma=1.5$. In the next step, we determined that:

$$
\begin{aligned}
& \gamma_{0}=1.5 \\
& \gamma_{1}=1.6666666666666667 \\
& \gamma_{2}=1.622222222222222 \\
& \gamma_{3}=1.61806970300121 \\
& \gamma_{4}=1.6180339913838113 \\
& \gamma_{5}=1.6180339887498947
\end{aligned}
$$

It is easy to see also for this case the great amount of precision in the fourth iteration, thus finding known $\gamma=1.6180$ as the gold number, or simply by symbology $\phi$. It is noteworthy that, except for the root equal to 1 , the remaining whole roots of the equation $(-0.6180$ and 1.6180$)$ are the same roots as the Fibonacci sequence, further establishing a similarity or numerical pattern of this sequence with the Fibonacci numbers.

Applying Newton's fractal with Google Colab, we have the root search shown in Figure 2.

Drawing a horizontal line in the previous figure, representing the $\mathrm{x}$-axis of the plane, shows that all lobes are located on it, diagnosing that the three roots are real. On the vertical line, y-axis, as noted by the absence of the lobes, it is shown that there are no complex roots for this Newton fractal. It is then noticed that the central lobe represents the root equal to 1, the larger lobe located on the left represents the negative root with a value of -0.61, and the right lobe represents the positive root called the gold number. The orange color in the central part of each lobe represents the exact value of the root, and the other colors around the lobes represent the number of iterations. Thus, the farther from the orange lobe, the farther it is from the root value of the function $f(\phi)=\phi^{3}-2 \phi^{2}+1$. 


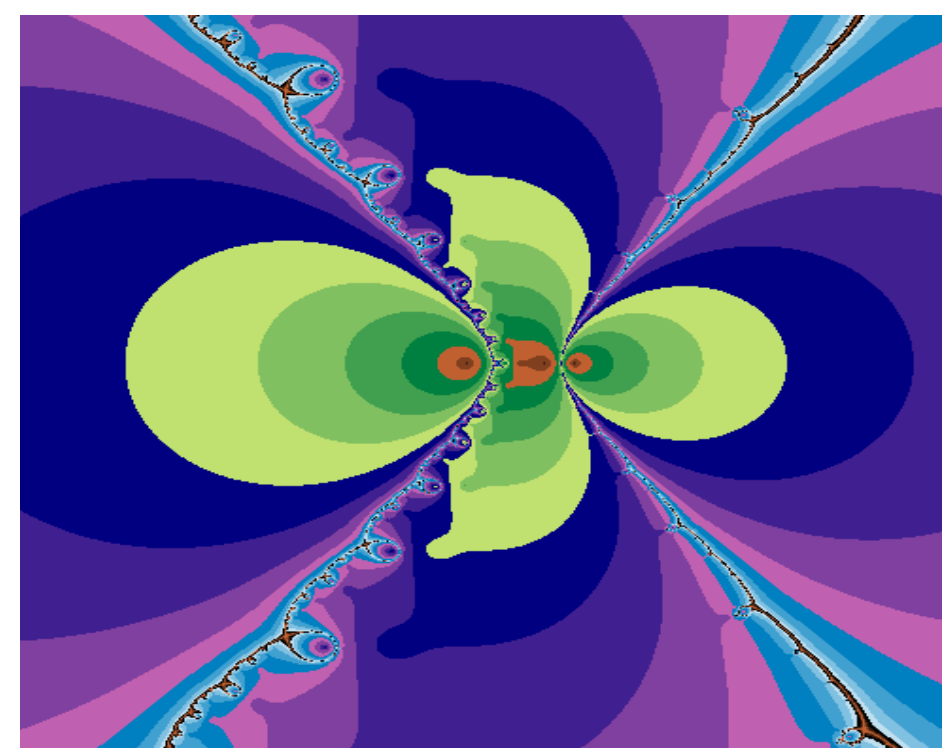

Figure 2. Newton's fractal of Leonardo's recurring sequence. Prepared by the authors

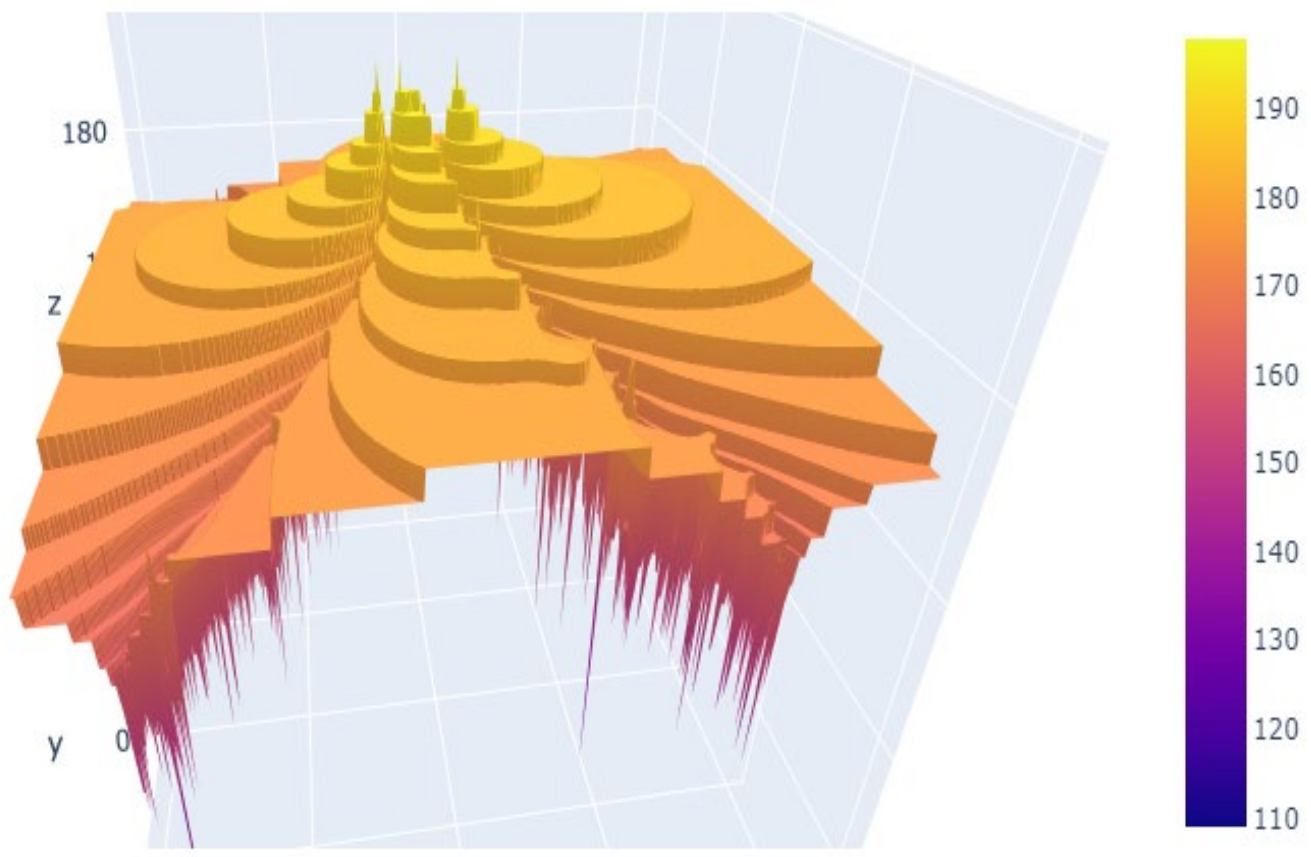

Figure 3. Leonardo's Newton Fractal in 3D. Prepared by the authors

According to Newton's method previously performed, we can see that five interactions were performed to find the roots. Thus, looking at Figure 2, we can see that there are eight color variations in each lobe, which is the maximum amount of iterations that exist until you find the root value that is located in the orange lobe.

The four traits shown in Figure 2 further show the root discontinuity, limiting which root will tend or approach depending on the choice of the initial numerical value. It is also important to highlight that the interval inserted in Phyton code to plot Newton's fractal was [-10,10], thus searching for the roots of the function that we indicate by. $f(\phi)=\phi^{3}-2 \phi^{2}+1$.

A 3D computational visualization for the Leonardo's Newton fractal is shown next in Figure 3, highlighting that the three roots are now represented by the mountains, where the higher up, the closer to the root value, and the more in the base, farther from the root value. The adjacent scale shows the proximity to the root, where the closer to the orange color, the closer it is to the root value. 


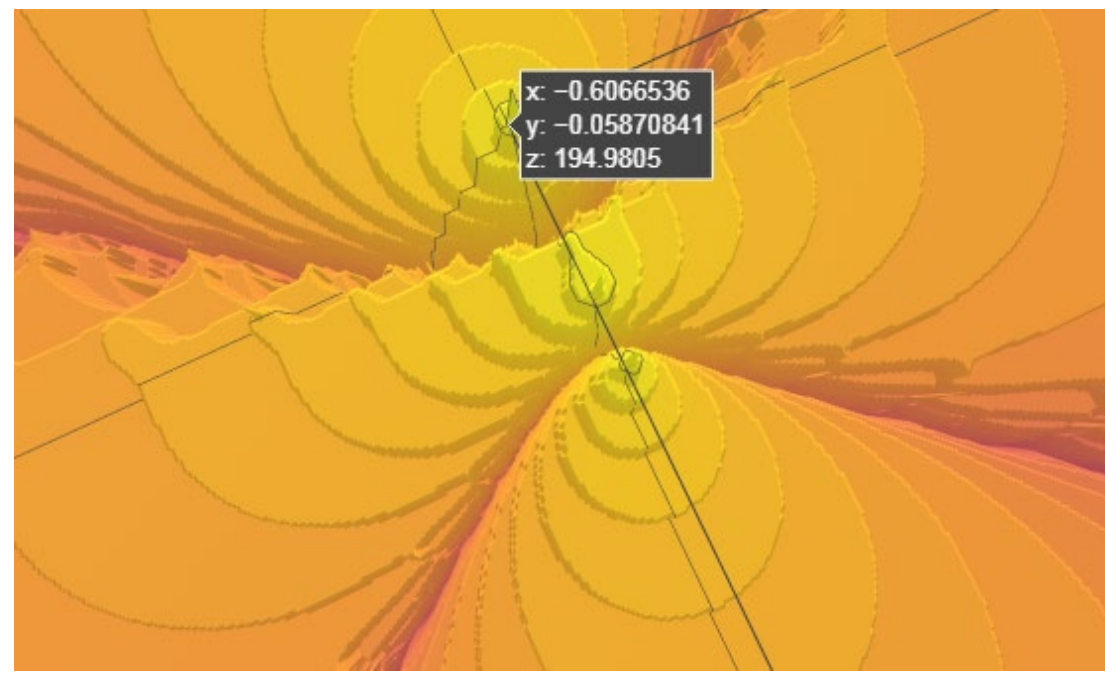

Figure 4. Visual analysis of Newton's fractal in 3D. Prepared by the authors

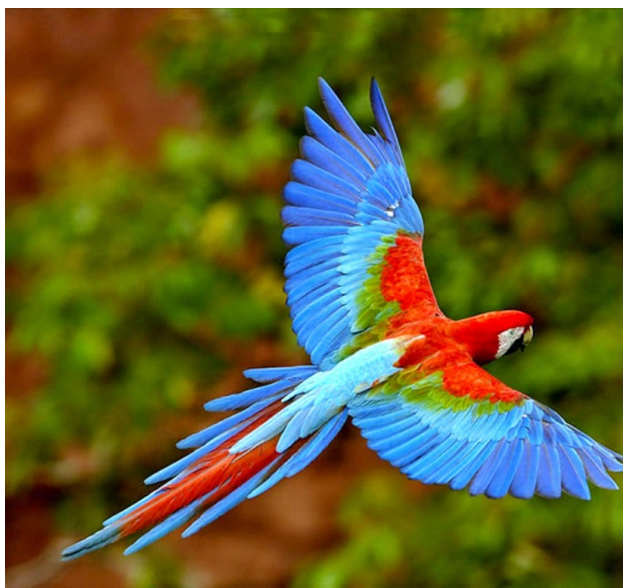

Figure 5. Bird in flight position and visual relations with a Fractal object. Source: Site SuperBichos

Given a more detailed visual analysis of Newton's fractal by zooming in, we can navigate with the mouse over the mountains, so the values are shown on the screen as shown in Figure 4.

From this perspective, we can see the existence of three axes, where the $\mathrm{x}$ and $\mathrm{y}$ axes show the values of the iterations to obtain the root. In the figure we have the value $\mathrm{x}=-0.6066536$ and $\mathrm{y}=-0.05870841$, where the value of $\mathrm{y}$ is very close to zero, with no imaginary part, whereas the real value of $\mathrm{x}$ is given by approximately -0.61 , validating the calculations shown for obtaining root previously.

In turn, the z-axis represents the proximity to the exact root value, wherein Figure 4 we have the value $\mathrm{z}$ $=194.9805$, where according to the scale shown in Figure 3, values above 190 are closer to the lobe orange, therefore closer to the correct numerical value.

Establishing a visual and geometrical relationship with objects found in nature, we notice a similarity of this Newton fractal of the Leonardo sequence, for example, with a bird (see Figure 5) at the moment of its flight, where its open arms are represented by the central lobe. Its head represented by the lobe of positive value referring to the number of another, and its lower limb as the root lobe of negative value.

There is also the "perfect photo" of a bird where an alcyon touches the water mirror with its beak, as shown in Figure 6. We note than another resemblance to Newton's fractal of this sequence of the 2D image seen by a other viewing angle. 


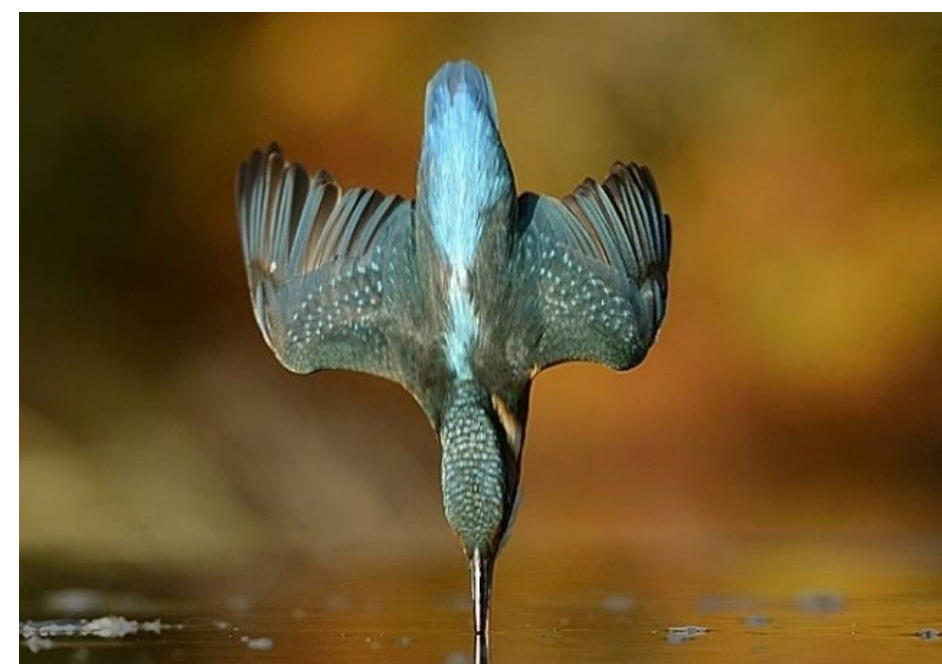

Figure 6. Bird's "perfect photo". Source: https://blogs.oglobo.globo.com/pagenotfound/post/apos-seis-anos-e720-mil-tentativas-fotografo-consegue-foto-perfeita-de-passaro.html

\section{CONCLUSION}

Leonardo's recurring sequence has recently been introduced in the scientific literature. We observe that the numbers derived from the set $\left\{L e_{n}\right\}_{n \in I N}$ are closely related to the Fibonacci sequence. Thus, having recurrence, we show its characteristic polynomial. Thus, it was possible to obtain the characteristic polynomial of the Leonardo sequence from its recurrence formula, to study and analyze its roots through the Newton method and Newton's fractal using Google Colab. This tool, being easy to handle, allowed to plot and visualize the fractals showing their visualization in the types discussed, so that the roots of this equation are then analyzed in a practical way.

Through the characteristic polynomial, it is possible to generalize these numbers to the integer field, as done in Santos and Alves (2017), expanding the research to future ones. It is also possible to extend this sequence, as done for Padovan numbers, for example, by finding some unexpected mathematical identities, as shown in Vieira and Alves (2019).

For future work, the development of Leonardo's quaternions and octonions is encouraged, as well as for Fibonacci in the work of Alves (2018). As done in this research, in which a similarity of Leonardo's Newton fractal generated with an object of nature was shown, this shows the connection of mathematics to other areas. Fractal-related studies are progressing more and more in health, especially for heartbeat investigations, so it is noticeable to realize the importance of analyzing fractals not only in mathematics, but also in several other areas (Felgueiras, 1996), thus stressing the importance of studying other methods applied to these fractals.

you:

\section{Disclosure statement}

No potential conflict of interest was reported by the authors.

\section{Notes on contributors}

Francisco Regis Vieira Alves - Federal Institute of Science and Technology of Ceara - IFCE, Brazil.

Renata Passos Machado Vieira - Federal Institute of Science and Technology of Ceara - IFCE, Brazil. 


\section{REFERENCES}

Alves, F. R. V. (2018). The Quaterniontonic and Octoniontonic Fibonacci Cassini's Identity: An Historical Investigation with the Maple's Help. International Eletronic Journal of Mathematics Education, 13(3), 125-138. https://doi.org/10.12973/iejme/2703

Alves, F. R. V., \& Catarino, P. M. M. C. Sequência matricial generalizada de Fibonacci e sequência matricial k-Pell: propriedades matriciais. C.Q.D. - Revista Eletrônica Paulista de Matemática, 15, 39-54. https://doi.org/10.21167/cqdvol15201923169664frvapmmec3954

Burton, B. (2009). Newton's method and fractais. Technical manuscript, Whitman College.

Carreira, A. S., \& Andrade, C. A. D. (1999). Geometria a várias dimensões - Fractais. Faculdade de Ciências, Universidade de Lisboa.

Catarino, P., \& Borges, A. (2019). On Leonardo numbers. Acta Mathematica Universitatis Comenianae, 1-12.

Cláudio, D. M., \& Martins, J. M. (2000). Cálculo Numérico Computacional, Teoria e Prática. São Paulo: Atlas.

Felgueiras, C. A. da S. (1996). Caracterização Fractal de Sequências de Batimentos Cardíacos Fetais (Master Thesis). Electrical and Computer Engineering, Faculdade de Engenharia da Universidade do Porto.

Lima, E. L. (1976). Curso de análise. Rio de Janeiro, Instituto de Matemática Pura e Aplicada. CNPq, 1.

Macedo, R. D. de. (2015). Introdução à Teoria de Fractais e à Derivada Métrica (Monograph of the Bachelor of Applied Mathematics course of the Institute of Mathematics, Statistics and Physics). Universidade Federal do Rio Grande.

Macleiod, A. J. (1984). A generalization of Newton-Raphson. Int. J. Math. Ed. Sci. Tech., 15(1), 117-120. https://doi.org/10.1080/0020739840150116

Oliveira, R. R. de, \& Alves, F. R. V. (2019). An investigation of the Bivariate Complex Fibonacci Polynomials supported in Didactic Engineering: an application of Theory of Didactics Situations (TSD). Acta Scientiae, 21(3), 170-195. https://doi.org/10.17648/acta.scientiae.v21iss3id3940

Randles, B. M., Pasquetto, I. V., Golshan, M. S., \& Borgman, C. L. (2017). Using the jupyter notebook as a tool for open science: An empirical study. ACM/IEEE Joint Conference on Digital Libraries (JCDL). IEEE, 1-2. https://doi.org/10.1109/JCDL.2017.7991618

Santos, A. A. dos, \& Alves, F. R. V. (2017). A fórmula de Binet como modelo de generalização e extensão da sequência de Fibonacci a outros conceitos matemáticos. C.Q.D. - Revista Eletrônica Paulista, 9, 4-22. https://doi.org/10.21167/cqdvol9201723169664aasfrva0422

Teodoro, M. M., \& Aguilar, J. C. Z. (2015). O método de Newton e fractais (Master's Dissertation in Mathematics), Federal University of São João del-Rei.

Vieira, R. P. M., \& Alves, F. R. V. (2019). Sequences of Tridovan and their identities. Notes on Number Theory and Discrete Mathematics, 25(3), 185-197, 2019. https://doi.org/10.7546/nntdm.2019.25.3.185-197

Zierler, N. (1959). Linear recurring sequences. Journal of the Society or Industrial and Applied Mathematics, 7(1), 31-48. https://doi.org/10.1137/0107003

http://www.iejme.com

http://www.iejme.com 